\title{
Residual Effects of THC via Novel Measures of Brain Perfusion and Metabolism in a Large Group of Chronic Cannabis Users
}

\author{
Francesca M Filbey, ${ }^{*, 1}$ Sina Aslan ${ }^{1,2}$, Hanzhang Lu $^{3}$ and Shin-Lei Peng ${ }^{3,4}$ \\ 'Center for BrainHealth, University of Texas at Dallas, Dallas, TX, USA; ${ }^{2}$ Advance MRI LLC, Frisco, TX, USA; ${ }^{3}$ Department of Radiology, Johns \\ Hopkins University, Baltimore, MD, USA; ${ }^{4}$ Department of Biomedical Imaging and Radiological Science, China Medical University, Taichung, Taiwan
}

\begin{abstract}
Given the known vascular effects of cannabis, this study examined the neurophysiological factors that may affect studies of brain activity in cannabis users. We conducted a systematic evaluation in $72 \mathrm{~h}$ abstinent, chronic cannabis users $(N=74)$ and nonusing controls $(N=10 \mathrm{I})$ to determine the association between prolonged cannabis use and the following neurophysiological indicators: $(\mathrm{I})$ global and regional resting cerebral blood flow (CBF), (2) oxygen extraction fraction (OEF), and (3) cerebral metabolic rate of oxygen $\left(\mathrm{CMRO}_{2}\right)$. We found that cannabis users had greater global $\mathrm{OEF}$ and $\mathrm{CMRO}_{2}$ compared with nonusers. Regionally, we found higher $\mathrm{CBF}$ in the right pallidum/ putamen of the cannabis users compared with nonusers. Global resting CBF and regional CBF of right superior frontal cortex correlated positively with creatinine-normalized $\Delta 9$-tetrahydrocannabinol (THC) levels. These findings demonstrate residual effects of cannabis use whereby global and regional brain metabolism are altered in those with prolonged cannabis exposure. These neurophysiological alterations should be considered in both research and clinical applications.
\end{abstract}

Neuropsychopharmacology (2018) 43, 700-707; doi:I0.I038/npp.2017.44; published online 22 March 2017

\section{INTRODUCTION}

Cannabis is the most commonly used illicit psychoactive drug, and therefore understanding its effects on behavior and brain is paramount. The primary psychoactive ingredient in cannabis, $\Delta$-9-tetrahydrocannabinol (THC), relaxes arterial walls resulting in lower blood pressure and increased blood flow to tissues. In the brain, THC binds to ubiquitous cannabinoid receptors (CB1) that are present in arterial tissue (Bilfinger et al, 1998) and regulate the microvascular environment via dose-dependent dilation of cerebral arterioles (Ellis et al, 1995). These cerebrovascular effects are variable across brain regions (Bloom et al, 1997; O'Leary et al, 2002), but have been most reported in frontal regions (Mathew and Wilson, 1993). These findings indicate that neurophysiological factors may contribute to cannabis' effects on brain activity, particularly as measured by the blood-oxygen-level-dependent (BOLD) signal.

To date, the effects of cannabis use on neurophysiological factors have primarily been measured using cerebral blood flow (CBF). In general, findings have been highly variable, perhaps because of the diverse and complex processes by which THC affects CBF including relaxation of vascular smooth muscle, alteration of partial pressure of carbon dioxide, and sympathetic stimulation (Zwillich et al, 1978). THC and CBF changes also have discordant time courses

\footnotetext{
* Correspondence: Dr FM Filbey, Center for BrainHealth, University of Texas at Dallas, 2200 West Mockingbird Lane, Dallas, TX 75235, USA, Tel: + 972883 33। I, E-mail: Francesca.Filbey@utdallas.edu

Received 12 October 2016; revised 6 February 2017; accepted 7 February 2017; accepted article preview online 27 February 2017
}

(Mathew et al, 2002). Neuroimaging studies have found differential effects of acute $v s$ chronic THC exposure. Specifically, acute exposure to cannabis has been associated with increased resting $\mathrm{CBF}$ in various brain regions including prefrontal areas and basal ganglia (Volkow et al, 1996), whereas chronic use has been associated with lower resting $\mathrm{CBF}$ in prefrontal and cerebellar metabolism as compared with nonusers (Volkow et al, 1996; Mathew and Wilson, 1991). CBF alterations have also been shown to be THC dose dependent (Mathew et al, 1989) and to normalize following abstinence (Jacobus et al, 2012). Altogether, these findings suggest that prolonged exposure to THC alters CBF that affects brain function and structure. At this time, however, the literature remains sparse with regard to how prolonged THC use may affect the brain in terms of blood oxygenation extraction and metabolism beyond CBF studies. This is of high relevance given the growing number of cannabis use recreationally and medically.

Given the known cerebrovascular effects of cannabis, this study examined putative residual effects of THC on neurophysiological parameters during rest. We measured global/regional CBF, oxygen extraction fraction (OEF), and cerebral metabolic rate of oxygen $\left(\mathrm{CMRO}_{2}\right)$ using several novel MRI techniques including pseudo-continuous arterial spin labeling (pCASL) MRI, T2-relaxation-under-spin-tagging (TRUST) MRI, and combined TRUST/phase contrast (PC) MRI, respectively. PC MRI estimates the total amount of blood flow delivered to the brain via bilateral internal carotid (ICA) and vertebral arteries (VA). PC MRI, however, does not provide regional $\mathrm{CBF}$ information. Thus, we used pCASL MRI to evaluate regional CBF. Next, we used TRUST 
MRI to first measure total brain's blood oxygenation $\left(Y_{\mathrm{v}}\right)$ and subsequently estimate oxygen extraction fraction and $\mathrm{CMRO}_{2}$. We investigated how the homeostasis among the aforementioned neurophysiological measures differed in $72 \mathrm{~h}$ abstinent, long-term cannabis users compared with nonusers. Furthermore, given the known CBF differences between males and females (Lu et al, 2011) as well as sex-dependent physiological and behavioral effects of THC (Ketcherside et al, 2016; Fattore and Fratta, 2010), we also explored potential group $\times$ sex interactions. Finally, we examined the association between the neurophysiological measures and patterns of cannabis use as well as cognitive functioning.

\section{MATERIALS AND METHODS}

\section{Participants}

A total of 175 participants, 74 cannabis users and 101 nonusers, were recruited for this study (see Table 1). Cannabis users were recruited based on self-reported history of regular cannabis use with a minimum of 5000 lifetime occasions of use in addition to daily use in the preceding 60 days before study enrollment. Verification of cannabis use was conducted via quantification of THC in urine via gas chromatography/mass spectrometry (GC/MS) normalized by urine creatinine $(\mathrm{Cr})$ concentration as per the recommendation by Huestis and Cone (1998). GC/MS was conducted by Quest Diagnostics (https://www.questdiagnostics.com/). The nonusing controls were recruited based on the absence of lifetime daily cannabis use. Exclusionary criteria for all participants were: history of neurological or psychiatric conditions, MR scanning contraindications, drug abuse, regular tobacco use ( $>1$ pack of cigarettes per month), left handedness, and non-native English speakers. As part of a larger study aimed to determine cue-elicited craving (see Filbey et al, 2016), cannabis users were scanned following a verified $72 \mathrm{~h}$ abstinence from cannabis. Abstinence was verified by self-report and decrease in $\mathrm{THC} / \mathrm{Cr}$ from baseline levels after the $72 \mathrm{~h}$ abstinence period. Those who were not able to comply with the abstinence requirement were excluded from this study.

Written informed consent was obtained from all participants in accordance with the institutional review board (IRB) of The University of Texas at Dallas and The University of Texas Southwestern Medical Center.

\section{Measures of Cannabis Use and IQ}

Duration of cannabis use, total number of lifetime occurrences, and THC/Cr levels measured at scan day (with $72 \mathrm{~h}$ abstinence) were collected as measures of cannabis use. We also collected the two-subtest version of the Wechsler Abbreviated Scale of Intelligence as a measure of general ability or IQ (Wechsler, 1999).

\section{MRI Acquisition}

MRI scans were performed on a 3 Tesla MR system (Philips Medical System, Best, The Netherlands). A body coil was used for radiofrequency (RF) transmission and an 8-channel head coil with parallel imaging capability was used for signal reception. We used several MRI techniques to investigate
Table I Participant Characteristics (Mean \pm SD)

\begin{tabular}{lccc}
\hline & Nonusers & Users & P-value \\
\hline$N$ & $10 \mid$ & 74 & - \\
Age & $30.3 \pm 10.3$ & $31.3 \pm 7.9$ & 0.45 \\
Gender (M/F) & $50 / 5 \mid$ & $46 / 28$ & 0.10 \\
Full IQ & $109.7 \pm 13.8$ & $106.7 \pm 12.0$ & 0.14 \\
Duration of use (years) & - & $10.6 \pm 7.3$ & - \\
No. of lifetime occurrence of & - & $14173.8 \pm 10866.0$ & - \\
cannabis use & & & \\
Baseline THC/Cr & - & $6.0 \pm 6.5$ & - \\
3-day abstinent THC/Cr (at & - & $1.9 \pm 1.4$ & - \\
time of scan) & - & $75.8 \pm 4.7$ & - \\
No. of hours since last cannabis & & & \\
use & & & \\
Number of participants per scan: & N (M/F) & & \\
$\quad$ PC MRI & $98(49 / 49)$ & $67(40 / 27)$ & \\
TRUST MRI & 93 (47/46) & $66(39 / 27)$ & \\
PCASL MRI & 94 (47/47) & $64(40 / 24)$ & \\
\hline
\end{tabular}

Abbreviations: M/F, male/female; IQ, Intelligence Quotient via Wechsler Abbreviated Scale of Intelligence as a measure of general intellect (Wechsler, 1999); THC, $\Delta$ 9-tetrahydrocannabinol; Cr, creatinine; PC MRI, phase contrast MRI to measure global cerebral blood flow; TRUST MRI, T2-relaxation-underspin-tagging MRI to measure global venous oxygenation; PCASL MRI, pseudocontinuous arterial spin labeling MRI to measure regional cerebral blood flow.

brain physiology at rest: a time-of-flight (TOF) angiogram to visualize the ICA and VA, PC MRI to measure whole-brain blood flow by positioning the imaging slab perpendicular to internal and vertebral arteries (Aslan et al, 2010b), TRUST MRI to assess blood oxygenation of the brain via superior sagittal sinus (Lu and Ge, 2008), and pCASL MRI sequence to measure regional blood flow of the brain (Aslan et al, 2010b). Finally, a high-resolution T1-weighted image was acquired as an anatomical reference.

The details of imaging parameters for each technique are as follow: the TOF angiogram imaging parameters were: TR/TE/ flip angle $=23 \mathrm{~ms} / 3.45 \mathrm{~ms} / 18^{\circ}$, field of view $(\mathrm{FOV})=160 \times$ $160 \times 70 \mathrm{~mm}^{3}$, voxel size $1.0 \times 1.0 \times 1.5 \mathrm{~mm}^{3}$, number of slices $=$ 47 , one saturation slab of $60 \mathrm{~mm}$ positioned above the imaging slab to suppress the venous vessels, duration $1 \mathrm{~min}$ and $26 \mathrm{~s}$. The slice of the PC velocity MRI was oriented perpendicular to the ICA and VA and the parameters were: single slice, voxel size $=0.50 \times 0.50 \mathrm{~mm}^{2}, \quad$ FOV $=200 \times 200 \mathrm{~mm}^{2}, \quad$ TR/TE $=20$ $17 \mathrm{msec}$, flip angle $=15^{\circ}$, slice thickness $=5 \mathrm{~mm}$, maximum velocity encoding $=80 \mathrm{~cm} / \mathrm{s}$, and scan duration $=30 \mathrm{~s}$. Global venous oxygenation, $Y_{\mathrm{v}}$, was noninvasively assessed from the superior sagittal sinus using a validated approach TRUST MRI (Lu and Ge, 2008; Lu et al, 2012; Xu et al, 2012). The TRUST MRI imaging parameters were: voxel size $3.44 \times 3.44 \times 5 \mathrm{~mm}^{3}$, $\mathrm{TR}=3000 \mathrm{~ms}$, $\mathrm{TI}=1022 \mathrm{~ms}$, four effective TEs: $0,40,80$, and $160 \mathrm{~ms}$, labeling thickness $100 \mathrm{~mm}$, gap $22.5 \mathrm{~mm}$, and scan duration $1.2 \mathrm{~min}$. Imaging parameters for pCASL experiments were: single-shot gradient-echo EPI, FOV $=240 \times 240$, matrix $=$ $80 \times 80$, voxel size $=3 \times 3 \mathrm{~mm}^{2}, 29$ slices acquired in ascending order, slice thickness $=5 \mathrm{~mm}$, no gap between slices, labeling duration $=1650 \mathrm{~ms}$, time interval between consecutive slice 
acquisitions $=35.5 \mathrm{~ms}, \mathrm{TR} / \mathrm{TE}=4211 / 14 \mathrm{~ms}$, SENSE factor 2.5 , number of controls/labels $=40$ pairs, $\mathrm{RF}$ duration $=0.5 \mathrm{~ms}$, pause between RF pulses $=0.5 \mathrm{~ms}$, labeling pulse flip angle $=18^{\circ}$, bandwidth $=2.7 \mathrm{kHz}$, echo train length $=35$, and scan duration $5.7 \mathrm{~min}$.

\section{MRI Analyses}

Global measures. Global CBF was calculated using PC MRI. Briefly, PC MRI provides a quantitative measurement of blood flow velocity in internal carotid artery and vertebral artery. The blood velocity was converted to flow rate by integrating over the cross-section of the vessels. Then, we divided the flow rate by the parenchyma intracranial mass (calculated from T1-weighted image and by assuming brain density of $\rho=1.06 \mathrm{~g} / \mathrm{ml}$ ) and obtained the whole-brain averaged blood flow in units of $\mathrm{ml} \mathrm{blood} / \mathrm{min} / 100 \mathrm{~g}$ brain (Aslan et al, 2010b).

A series of steps were carried out to determine total brain oxygen extraction and cerebral oxygen metabolism. First, TRUST MRI was utilized to noninvasively obtain global venous oxygenation, $Y_{\mathrm{v}}$, from the superior sagittal sinus (Liu et al, 2012; Lu and Ge, 2008; Xu et al, 2012). Briefly, TRUST data were processed by subtracting control and label images to yield pure venous blood signal. Then, the venous blood signals were fitted to a monoexponential function to obtain T2 that was in turn converted to $Y_{\mathrm{v}}$ (ie global venous oxygenation) via a calibration plot ( $\mathrm{Lu}$ et al, 2012). Finally, total $\mathrm{CMRO}_{2}$ was then estimated from global cerebral blood flow and $Y_{\mathrm{v}}$ based on Fick's principle in units of $\mu \mathrm{mol}$ $\mathrm{O}_{2} / 100 \mathrm{~g} / \mathrm{min}$ (Kety and Schmidt, 1948; Peng et al, 2014; Xu et al, 2009):

$$
C M R O_{2}=t C B F \cdot\left(Y_{\mathrm{a}}-Y_{\mathrm{v}}\right) \cdot C_{\mathrm{h}}
$$

where $C M R O_{2}$ and $t C B F$ are total $\mathrm{CMRO}_{2}$ and cerebral blood flow, respectively; $Y_{\mathrm{a}}$ and $Y_{\mathrm{v}}$ are oxygen saturation percentage in arterial and venous blood, respectively; and $C_{\mathrm{h}}$ is a constant representing the capacity of blood to carry $\mathrm{O}_{2}$ and is well established in physiology literature (Guyton and Hall, 2005). Both $Y_{\mathrm{a}}$ and $C_{\mathrm{h}}$ were estimated based on prior findings and were adjusted for age and sex (Peng et al, 2014). In addition, we estimated the OEF by calculating the difference between arterial blood oxygenation and global venous oxygenation in sagittal sinus: $\left(Y_{\mathrm{a}}-Y_{\mathrm{v}}\right) / Y_{\mathrm{a}}$.

Regional measures. In terms of regional brain blood flow, we analyzed pCASL MRI data using routine processing (Aslan et al, 2010b). The pCASL image series were realigned to the first volume for motion correction (SPM5's realign function, University College London, UK). ASL images with motion of $>3 \mathrm{~mm}$ and $>3^{\circ}$ were excluded from further processing. Next, an in-house MATLAB (Mathworks, Natick, MA) program was used to calculate the difference between averaged control and label images. Then, the difference image was corrected for imaging slice delay time to yield CBF-weight image that was normalized to the Montreal Neurological Institute (MNI) brain template. Finally, the absolute CBF ( $\mathrm{aCBF}$ ) was estimated in the units of $\mathrm{ml} \mathrm{blood} / \mathrm{min} / 100 \mathrm{~g}$ of brain tissue. The voxel-wise analyses were performed on the relative CBF ( $\mathrm{rCBF}$ ) maps that included dividing the aCBF spatial maps by the whole-brain absolute CBF value. In a prior publication, we showed that such technique improves the sensitivity of regional differences by reducing physiological variations (Aslan and Lu, 2010a). During voxel-based analyses (VBAs), the individual $\mathrm{rCBF}$ maps were spatially smoothed (with full-width half-maximum (FWHM) of $4 \mathrm{~mm}$ ) to account for small differences in sulci/gyri location across subjects. To define a threshold for the VBA results, we used 3dClustsim (with -acf option) in AFNI (NIMH Scientific and Statistical Computing Core, Bethesda, MD) that controls for false positive activation clusters over the set of all activation clusters throughout the whole-brain volume given voxel size, whole-brain volume, and effective smoothness (inherent smoothness plus additional smoothness applied). We refer to this procedure in the Results section as cluster-level familywise error correction (FWE corrected). The error correction was conditional on three criteria: smoothness of the voxel map, a magnitude threshold for defining clusters of contiguous voxels, and a minimum volume for each cluster. For the CBF VBA, we used $p$ (FWE corrected) value of $<0.05$ that is based on an effective smoothness of $8.7 \mathrm{~mm}$ FWHM, a cluster-defining magnitude corresponding to the 99.5th percentile of $t$-statistic distribution at $p<0.0002$, and a minimum volume of 34 voxels $\left(272 \mathrm{~mm}^{3}\right)$.

\section{Statistical Analyses}

A general linear model (GLM) was applied to assess the effects of cannabis on the neurophysiological brain measures (ie, $\mathrm{CBF}, \mathrm{OEF}$, and $\mathrm{CMRO}_{2}$ ). For each of these dependent variables, the model included group status (user, nonuser), sex (male, female), and group $\times$ sex interaction. Finally, voxel-wise correlation analyses were conducted in the cannabis users to determine the relationship between neurophysiological indexes and duration of cannabis use, total number of lifetime occurrences, scan day $\mathrm{THC} / \mathrm{Cr}$, and IQ. A statistical threshold of $p$ (FWE corrected) $<0.05$ and cluster size of $>272 \mathrm{~mm}^{3}$ was applied for these analyses.

\section{RESULTS}

\section{Participant Characteristics}

Only cannabis users who (1) tested positive for THC at baseline and (2) had a decrease in THC at the time of scan were included in this study. Of the 74 cannabis users, one had an incomplete scan and was excluded from further analyses. For each imaging scan, there were varying number of participants with high motion and were subsequently excluded from respective analyses. Table 1 summarizes the number of participants included per analysis. There were no significant differences in age, gender, and IQ $(p>0.05)$. Of the nonusers, 73 reported alcohol use and 2 reported regular tobacco use. Of the nonusers, 63 reported alcohol use, and 21 of the users reported regular tobacco use.

\section{Global Neurophysiological Markers}

Table 2 summarizes the mean global neurophysiological measures and Table 3 lists the statistical results for the factorial analysis. 
Table 2 Global Brain Measurements (Mean \pm SEM)

\begin{tabular}{lcc}
\hline & Nonusers & Users \\
\hline CBF $(\mathrm{m} / / \mathrm{min} / \mathrm{l} 00 \mathrm{~g})$ & \\
All & $59.9 \pm 9.8$ & $57.8 \pm 9.2$ \\
Males & $56.8 \pm 9.5$ & $55.2 \pm 7.7$ \\
Females & $62.9 \pm 9.8$ & $61.7 \pm 10.0$ \\
& & \\
OEF (\%) & & $35.0 \pm 7.3$ \\
All & $32.5 \pm 7.6$ & $35.1 \pm 7.6$ \\
Males & $31.1 \pm 7.2$ & $34.8 \pm 7.0$ \\
Females & $33.9 \pm 7.8$ & \\
& & $162.2 \pm 25.7$ \\
CMRO & & \\
All & & $158.3 \pm 25.7$ \\
Males & $167.8 \pm 25.3$ \\
Females & $100 \mathrm{~g} / \mathrm{min})$ & \\
\hline
\end{tabular}

Table 3 Statistical Test Results for Global Neurophysiological Measures Are Shown

\begin{tabular}{lcc}
\hline & F-value & $P$-value \\
\hline CBF $(\mathrm{m} / / \mathrm{min} / \mathrm{l00} g)$ & $F(1,|6|)=0.20$ & \\
Group & $F(1,|6|)=18.80$ & $<.66$ \\
Sex & $F(1,|6|)=0.01$ & $0.00 \mid$ \\
Group $\times$ sex &
\end{tabular}

$\begin{array}{lll}\text { OEF (\%) } & & \\ \text { Group } & F(I, I 55)=4.69 & 0.03 \\ \text { Sex } & F(I, 155)=0.99 & 0.32 \\ \text { Group x sex } & F(I, 155)=1.73 & 0.19\end{array}$

$\mathrm{CMRO}_{2}(\mu \mathrm{mol} / \mathrm{l} 00 \mathrm{~g} / \mathrm{min})$

$\begin{array}{lcc}\text { Group } & F(I, \mid 55)=4.20 & 0.04 \\ \text { Sex } & F(I, \mid 55)=12.84 & <0.00 I \\ \text { Group x sex } & F(I, \mid 55)=1.66 & 0.20\end{array}$

Abbreviations: $\mathrm{CBF}$, cerebral blood flow; OEF, oxygenation extraction fraction; $\mathrm{CMRO}_{2}$, cerebral metabolic rate of oxygen.

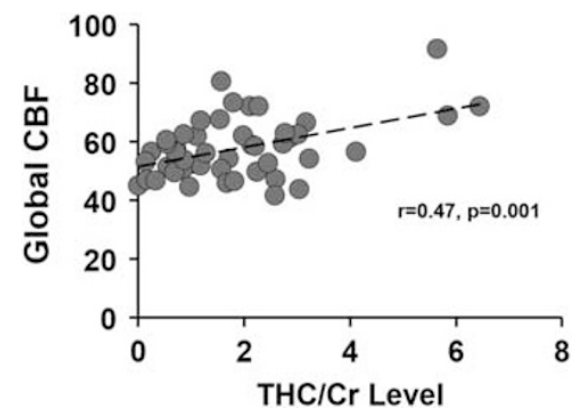

Figure I Scatterplot of global cerebral blood flow (CBF) and $\mathrm{THC} / \mathrm{Cr}$ in cannabis users. A significant correlation was found between $\mathrm{CBF}$ and $\mathrm{THC} / \mathrm{Cr}$ level $(r=0.47$ and $p=0.00 \mathrm{I})$.
$C B F$. There was no significant global CBF effect for group $(\mathrm{F}(1,161)=0.20, \quad p=0.66)$ or group $\times \operatorname{sex}$ interaction $(\mathrm{F}(1,161)=0.01, p=0.91)$. As expected, there was an effect of sex indicating higher CBF in females compared with males $(\mathrm{F}(1,161)=18.80, p<0.001)(\mathrm{Lu}$ et al, 2011).

OEF. We found a significant group effect in total OEF. Users had higher OEF compared with nonusers $(F(1,155)=4.69, p=0.03)$. There was no significant effect of $\operatorname{sex}(F(1,155)=0.99, p=0.32)$ or group $\times$ sex interaction $(\mathrm{F}(1,155)=1.73, p=0.19)$ in OEF.

$\mathrm{CMRO}_{2}$. We found a significant group effect in $\mathrm{CMRO}_{2}$. Users had higher $\mathrm{CMRO}_{2}$ compared with controls $(\mathrm{F}(1,155)=4.20, p=0.04)$. We also found that females have higher $\mathrm{CMRO}_{2}$ compared with males $(\mathrm{F}(1,155)=12.84$, $p<0.001)$. There was no group $\times$ sex interaction effect in $\mathrm{CMRO}_{2}(\mathrm{~F}(1,155)=1.66, p=0.20)$.

$C B F$ vs OEF. We conducted a confirmatory analysis to verify the coupling between global CBF and OEF. Our results paralleled the findings of Peng et al (2014) of an inverse correlation in CBF and OEF in both the user $(r=-0.58$ and $p<0.001)$ and nonuser group $(r=-0.58$ and $p<0.001)$.

Correlation analyses. As shown in Figure 1, a significantly positive association emerged in the users between resting global CBF and THC/Cr $(r=0.47$ and $p=0.001)$. No significant correlations were found between global neurophysiological markers and duration of cannabis use, total number of lifetime occurrences, or IQ.

\section{Regional Neurophysiological Markers}

Figure 2 and Table 4 summarize the results of voxel-based analyses. First, users had significantly higher blood flow in the right pallidum and putamen compared with nonusers: peak MNI $(+20-4-2), F(1,154)=27.05$, cluster size $=$ $384 \mathrm{~mm}^{3}$ at $p$ (FWE-corrected $)<0.05$, and $k \geqslant 272 \mathrm{~mm}^{3}$ (Figure 2). Second, we found a significant effect of sex: males had a higher regional $\mathrm{CBF}$ in the right insula compared with females whereas females had a higher CBF in the left posterior cingulate and bilateral precuneus compared with males. No significant interaction between group and sex was found.

Correlation analyses. Voxel-wise correlation analysis showed a positive correlation between scan day $\mathrm{THC} / \mathrm{Cr}$ level and the CBF of right superior frontal cortex at peak MNI coordinates: $(+20+48+18), t$-score of 4.45 , cluster size $=328 \mathrm{~mm}^{3}, p(\mathrm{FWE}$ corrected $)<0.05$, and cluster size $\geqslant 272 \mathrm{~mm}^{3}$ (Figure 3). No significant correlations were found between regional neurophysiological markers and duration of cannabis use, total number of lifetime occurrences, or IQ.

\section{DISCUSSION}

In this study, we evaluated neurophysiological indicators via novel measures of brain perfusion and metabolism in a large and well-characterized group of $72 \mathrm{~h}$ abstinent cannabis 


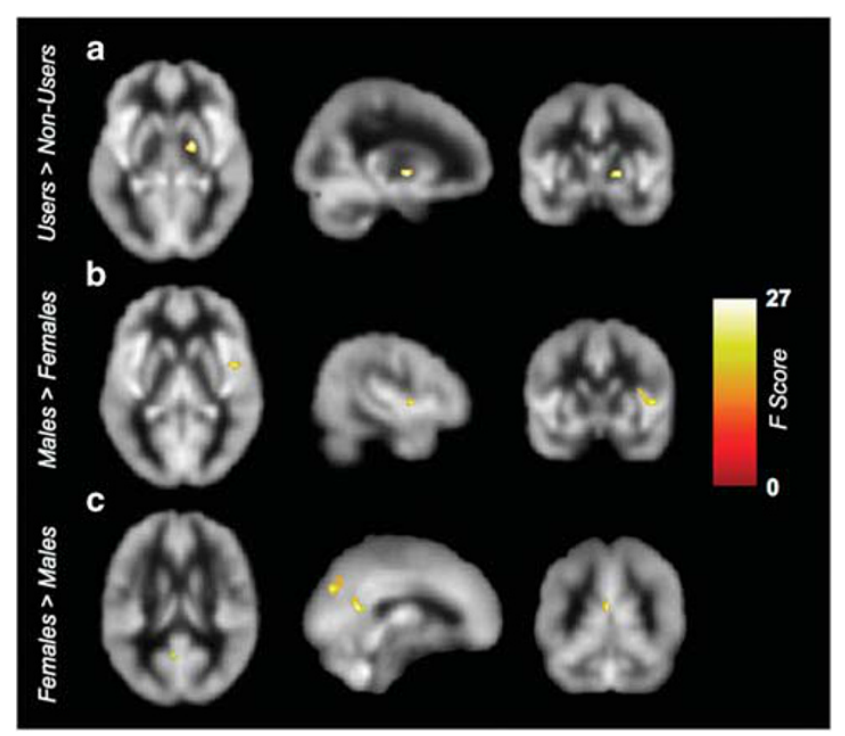

Figure 2 Results of cerebral blood flow (CBF) voxel-based comparisons superimposed on average CBF map of all participants. (a) The cannabis users showed higher CBF in right pallidum compared with nonusers. (b) Males showed higher CBF in the right insula, whereas (c) females showed higher $\mathrm{CBF}$ in the right posterior cingulate cortex and bilateral precuneus (FWE cluster level corrected at $p<0.05$ ).

Table 4 Regional CBF Differences at Rest between Users and Nonusers

\begin{tabular}{|c|c|c|c|c|c|c|}
\hline \multirow[b]{2}{*}{ Brain regions } & \multirow[b]{2}{*}{ BA } & \multirow[b]{2}{*}{$\begin{array}{l}\text { Cluster size } \\
\left(\mathrm{mm}^{3}\right)\end{array}$} & \multicolumn{3}{|c|}{ MNI } & \multirow[b]{2}{*}{ F-score } \\
\hline & & & $\mathbf{x}$ & $\mathbf{Y}$ & $\mathbf{Z}$ & \\
\hline \multicolumn{7}{|l|}{ Main effect of group } \\
\hline \multicolumn{7}{|l|}{ Users $>$ Nonusers } \\
\hline Right pallidum & - & 384 & 20 & -4 & -2 & 27.05 \\
\hline \multicolumn{7}{|l|}{ Main effect of sex } \\
\hline \multicolumn{7}{|l|}{ Males $>$ females } \\
\hline Right insula & 13 & 720 & 48 & 2 & 4 & 23.05 \\
\hline \multicolumn{7}{|l|}{ Females $>$ males } \\
\hline $\begin{array}{l}\text { Left posterior } \\
\text { cingulate cortex }\end{array}$ & 23 & 360 & -4 & -56 & 16 & 26.50 \\
\hline $\begin{array}{l}\text { Left/right } \\
\text { precuneus }\end{array}$ & $7 / 19$ & 1288 & -10 & -78 & 48 & 25.89 \\
\hline
\end{tabular}

Group $\times$ sex interaction

No significant difference

FWE cluster level, corrected $p<0.05$, voxel threshold at $p=0.0002$ and $K=272 \mathrm{~mm}^{3}$.

users relative to nonusers. We found greater regional CBF in the right pallidum/putamen in users compared with nonusers, in line with reported functional and structural changes in this critical region in long-term cannabis users (Filbey et al, 2014). These findings of regional but not global CBF differences between users and nonusers are concordant with region-specific effects described in studies of both chronic (Bloom et al, 1997; Mathew and Wilson, 1993) and acute effects of cannabis use (O'Leary et al, 2002). Increases in resting $\mathrm{CBF}$ in basal ganglia structures have been linked to elevated salience and motivation processes that are mechanisms that contribute to compulsive use of cannabis (Volkow et al, 1996). The pallidum plays a critical role as the interface between the ventral striatum and the thalamus with the ventral pallidum, specifically, mediating reward and motivation processes (Smith et al, 2009). The putamen is dense with cannabinoid receptors (Miller and Walker, 1996) and has been shown to have functional and structural alterations that are associated with cannabis use (Filbey et al, 2016; Wetherill et al, 2015; Yip et al, 2014). Because THC relaxes arterial walls and increases blood flow to tissues, our finding adds to the literature by demonstrating that the pathomechanism underlying the reported functional and structural alterations in the pallidum/putamen may be due to altered perfusion that is closely coupled with neural activity.

Notably, other studies have reported decreases rather than increases in $\mathrm{CBF}$ in chronic cannabis users (Martin-Santos et al, 2010; Mathew and Wilson, 1991; Lundqvist et al, 2001). The discrepancy with existing reports may be because of heterogeneity both within and between study samples. For example, Mathew et al (1989) reported dissociable CBF effects where experienced users had greater CBF whereas inexperienced users had decreased CBF following THC exposure. Length of abstinence can also affect CBF effects. Tunving et al (1986) noted changes during the course of abstinence whereby $\mathrm{rCBF}$ level in cannabis users was significantly $(11 \%)$ lower than that of age- and sexmatched healthy controls, although (12\%) increases after 9-60 days of detoxification of the CBF level was noted at follow-up. Given that our sample of cannabis users were heavy, long-term users and that data were collected following 3 days of required abstinence, this observation may be an 'overshoot of recovery' of neurophysiological function during the early stages of abstinence in chronic cannabis users. In other words, these findings reflect the residual effects of both long-term cannabis exposure and adaptive changes that occur after a short period of abstinence. Future studies should measure the trajectory of these effects to fully determine whether this phenomenon is indeed a recovery of typical function.

It is also important to note that in addition to chronicity and cessation from cannabis use that complicate the understanding of THC effects on CBF, individual variation in response to THC also plays a role (Volkow et al, 1991). One important individual factor to consider is sex-specific effects of cannabis (see review by Ketcherside et al, 2016). Although we found concordant findings of a main effect of sex on CBF and $\mathrm{CMRO}_{2}$ where females had greater $\mathrm{CBF}$ and $\mathrm{CMRO}_{2}$ (Lu et al, 2011), we did not find a group $\times$ sex interaction in any of the brain measures. This could be due, in part, to the smaller number of females in the cannabis using group. Another possibility for the absence of a group $\times$ sex effect may be that any increased neuronal needs reflected by greater $\mathrm{OEF} / \mathrm{CMRO}_{2}$ and $\mathrm{CBF}$ in the pallidum/putamen in the cannabis users relative to nonusers may be compensated for by the typically elevated global CBF and $\mathrm{CMRO}_{2}$ in females. Greater global $\mathrm{CBF}$ and $\mathrm{CMRO}_{2}$ in 

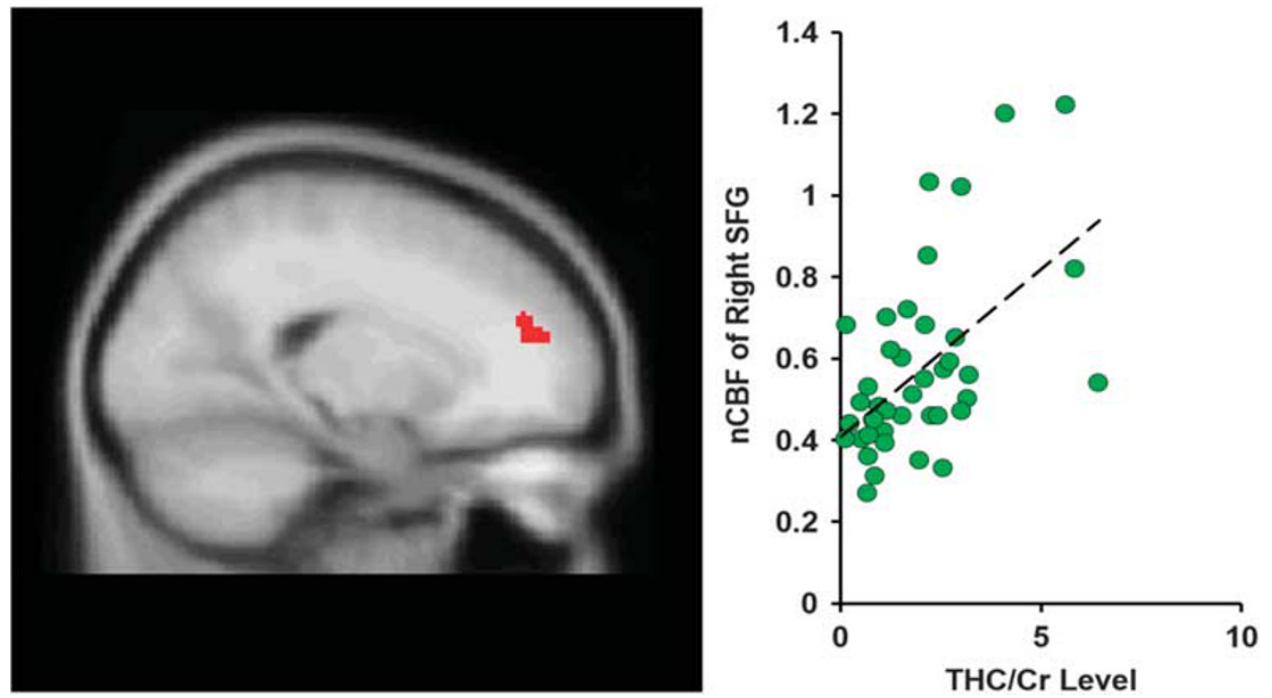

Figure 3 The cannabis users showed a significantly positive association between right superior frontal cortex cerebral blood flow and THC/Cr level $(p<0.05$, FWE corrected).

females have been suggested to reflect hormonal, muscle, and tissue content differences between sexes (Fattore and Fratta, 2010).

We found positive associations between global and regional $\mathrm{CBF}$ and $\mathrm{THC}$ metabolite levels that further support the relationship between changes in blood flow and exposure to cannabis (Mathew et al, 1997; Volkow et al, 1996). Alterations in the superior frontal gyrus have previously been reported in cannabis users in terms of reduced thickness (Lopez-Larson et al, 2011) and enhanced activation during memory and inhibitory tasks (Smith et al, 2010; Tapert et al, 2007). This finding highlights the differential effects of acute and prolonged THC exposure that brings neural adaptations in long-term cannabis users to bear.

These findings have several implications. First, because $\mathrm{CBF}$ and brain function are closely coupled, it is likely that widely observed changes in brain response (eg, BOLD fMRI) may be partly explained by these latent cerebrovascular changes. There is emergent literature that has begun to shed light on the interplay between neural and vascular components in substance use disorders. Similar investigations have linked changes in EEG patterns with cerebral perfusion deficits in long-term cannabis users during abstinence that were interpreted as under-arousal of these systems (Herning et al, 2008). Using $\mathrm{CMRO}_{2}$, long-term cocaine users have also been shown to have reduced $\mathrm{CMRO}_{2}$ relative to nonusing controls that was associated with lifetime frequency of cocaine use (Liu et al, 2014).

It is worth noting that at our selected threshold, no group effects emerged in cortical areas. However, a less conservative threshold of $p<0.01$ and $K>200$ revealed that nonusers had higher regional $\mathrm{CBF}$ in cortical areas including right middle temporal, left middle frontal, and right superior frontal cortices compared with users. Thus, although differences were observed in other regions, our findings suggest that neurophysiological changes as a result of cannabis use are most robust in the pallidum/putamen area. Indeed, structural and functional alterations in the putamen have been reported to be a risk factor for cannabis use disorder (Wetherill et al, 2015), predict development of problems related to cannabis use (Vingerhoets et al, 2016), and are related to treatment success (Yip et al, 2014).

\section{LIMITATIONS AND CONCLUSIONS}

Given the unique effects of $\mathrm{CBF}$ and THC on sex, it was surprising that we did not find a group $\times$ sex interaction. It is possible that our unequal sample sizes, which was because of smaller number of females in the users group, contributed to the failure to find an effect. Future studies should focus on obtaining equal sample sizes across group and sex. Although we found that $\mathrm{CBF}$ was associated with $\mathrm{THC} / \mathrm{Cr}$, no other association between measures of cannabis use or IQ emerged. Future studies should consider evaluating other measures of cognitive function related to cannabis use, such as inhibitory control or reward sensitivity. Finally, interpretation of these findings is limited by the cross-sectional nature of this study. Thus, we cannot infer causality in these effects. To conclude, cerebrovascular markers are promising for the assessment of the long-term effects of cannabis use on the brain. Understanding these neurophysiological mechanisms in cannabis users and their interplay with other mechanisms related to cannabis use will inform the widely reported alterations in brain structure and function in this population.

\section{FUNDING AND DISCLOSURE}

This research was funded by NIH R01 DA030344 (to FMF) and NIH R01 MH084021 (to HL). The authors declare no conflict of interest.

\section{ACKNOWLEDGMENTS}

We are grateful to the Filbey lab research assistants, MR technologists at UTSW Advance Imaging Research Center, and to all participants who took part in this study. 


\section{REFERENCES}

Aslan S, Lu H (2010a). On the sensitivity of ASL MRI in detecting regional differences in cerebral blood flow. Magn Reson Imaging 28: 928-935.

Aslan S, Xu F, Wang PL, Uh J, Yezhuvath US, van Osch M et al (2010b). Estimation of labeling efficiency in pseudocontinuous arterial spin labeling. Magn Reson Med 63: 765-771.

Bilfinger TV, Salzet M, Fimiani C, Deutsch DG, Tramu G, Stefano GB (1998). Pharmacological evidence for anandamide amidase in human cardiac and vascular tissues. Int J Cardiol 64 (Suppl 1): S15-S22.

Bloom AS, Tershner S, Fuller SA, Stein EA (1997). Cannabinoidinduced alterations in regional cerebral blood flow in the rat. Pharmacol Biochem Behav 57: 625-631.

Ellis EF, Moore SF, Willoughby KA (1995). Anandamide and delta 9-THC dilation of cerebral arterioles is blocked by indomethacin. Am J Physiol 269(6 Pt 2): H1859-H1864.

Fattore L, Fratta W (2010). How important are sex differences in cannabinoid action? Br J Pharmacol 160: 544-548.

Filbey FM, Aslan S, Calhoun VD, Spence JS, Damaraju E, Caprihan A et al (2014). Long-term effects of marijuana use on the brain. Proc Natl Acad Sci USA 111: 16913-16918.

Filbey FM, Dunlop J, Ketcherside A, Baine J, Rhinehardt T, Kuhn B et al (2016). fMRI study of neural sensitization to hedonic stimuli in long-term, daily cannabis users. Hum Brain Mapp 37: 3431-3443.

Guyton A, Hall J (2005). Respiration. Saunders, Elsevier: Philadelphia, PA.

Herning RI, Better W, Cadet JL (2008). EEG of chronic marijuana users during abstinence: relationship to years of marijuana use, cerebral blood flow and thyroid function. Clin Neurophysiol 119: 321-331.

Huestis MA, Cone EJ (1998). Urinary excretion half-life of 11-nor9-carboxy-delta9-tetrahydrocannabinol in humans. Ther Drug Monit 20: 570-576.

Jacobus J, Goldenberg D, Wierenga CE, Tolentino NJ, Liu TT, Tapert SF (2012). Altered cerebral blood flow and neurocognitive correlates in adolescent cannabis users. Psychopharmacology (Berl) 222: 675-684.

Ketcherside A, Baine J, Filbey F (2016). Sex effects of marijuana on brain structure and function. Curr Addict Rep 3: 323-331.

Kety SS, Schmidt CF (1948). The nitrous oxide method for the quantitative determination of cerebral blood flow in man: theory, procedure and normal values. J Clin Invest 27: 476-483.

Liu P, Lu H, Filbey FM, Tamminga CA, Cao Y, Adinoff B (2014). MRI assessment of cerebral oxygen metabolism in cocaineaddicted individuals: hypoactivity and dose dependence. NMR Biomed 27: 726-732.

Liu P, Uh J, Devous MD, Adinoff B, Lu H (2012). Comparison of relative cerebral blood flow maps using pseudo-continuous arterial spin labeling and single photon emission computed tomography. NMR Biomed 25: 779-786.

Lopez-Larson MP, Bogorodzki P, Rogowska J, McGlade E, King JB, Terry J et al (2011). Altered prefrontal and insular cortical thickness in adolescent marijuana users. Behav Brain Res 220: 164-172.

Lu H, Ge Y (2008). Quantitative evaluation of oxygenation in venous vessels using T2-Relaxation-Under-Spin-Tagging MRI. Magn Reson Med 60: 357-363.

Lu H, Xu F, Grgac K, Liu P, Qin Q, van Zijl P (2012). Calibration and validation of TRUST MRI for the estimation of cerebral blood oxygenation. Magn Reson Med 67: 42-49.

Lu H, Xu F, Rodrigue KM, Kennedy KM, Cheng Y, Flicker B et al (2011). Alterations in cerebral metabolic rate and blood supply across the adult lifespan. Cereb Cortex 21: 1426-1434.
Lundqvist T, Jonsson S, Warkentin S (2001). Frontal lobe dysfunction in long-term cannabis users. Neurotoxicol Teratol 23: 437-443.

Martin-Santos R, Fagundo AB, Crippa JA, Atakan Z, Bhattacharyya S, Allen P et al (2010). Neuroimaging in cannabis use: a systematic review of the literature. Psychol Med 40: 383-398.

Mathew RJ, Wilson WH (1991). Substance abuse and cerebral blood flow. Am J Psychiatry 148: 292-305.

Mathew RJ, Wilson WH (1993). Acute changes in cerebral blood flow after smoking marijuana. Life Sci 52: 757-767.

Mathew RJ, Wilson WH, Coleman RE, Turkington TG, DeGrado TR (1997). Marijuana intoxication and brain activation in marijuana smokers. Life Sci 60: 2075-2089.

Mathew RJ, Wilson WH, Tant SR (1989). Acute changes in cerebral blood flow associated with marijuana smoking. Acta Psychiatr Scand 79: 118-128.

Mathew RJ, Wilson WH, Turkington TG, Hawk TC, Coleman RE, DeGrado TR et al (2002). Time course of tetrahydrocannabinol-induced changes in regional cerebral blood flow measured with positron emission tomography. Psychiatry Res 116: 173-185.

Miller AS, Walker JM (1996). Electrophysiological effects of a cannabinoid on neural activity in the globus pallidus. Eur $J$ Pharmacol 304: 29-35.

O'Leary DS, Block RI, Koeppel JA, Flaum M, Schultz SK, Andreasen NC et al (2002). Effects of smoking marijuana on brain perfusion and cognition. Neuropsychopharmacology 26: 802-816.

Peng SL, Dumas JA, Park DC, Liu P, Filbey FM, McAdams CJ et al (2014). Age-related increase of resting metabolic rate in the human brain. Neuroimage 98: 176-183.

Smith AM, Longo CA, Fried PA, Hogan MJ, Cameron I (2010). Effects of marijuana on visuospatial working memory: an fMRI study in young adults. Psychopharmacology (Berl) 210: 429-438.

Smith KS, Tindell AJ, Aldridge JW, Berridge KC (2009). Ventral pallidum roles in reward and motivation. Behav Brain Res 196: 155-167.

Tapert SF, Schweinsburg AD, Drummond SP, Paulus MP, Brown SA, Yang TT et al (2007). Functional MRI of inhibitory processing in abstinent adolescent marijuana users. Psychopharmacology (Berl) 194: 173-183.

Tunving K, Thulin SO, Risberg J, Warkentin S (1986). Regional cerebral blood flow in long-term heavy cannabis use. Psychiatry Res 17: 15-21.

Vingerhoets WA, Koenders L, van den Brink W, Wiers RW, Goudriaan AE, van Amelsvoort $\mathrm{T}$ et al (2016). Cue-induced striatal activity in frequent cannabis users independently predicts cannabis problem severity three years later. J Psychopharmacol 30: 152-158.

Volkow ND, Gillespie H, Mullani N, Tancredi L, Grant C, Ivanovic $M$ et al (1991). Cerebellar metabolic activation by delta-9-tetrahydro-cannabinol in human brain: a study with positron emission tomography and 18F-2-fluoro-2-deoxyglucose. Psychiatry Res 40: 69-78.

Volkow ND, Gillespie H, Mullani N, Tancredi L, Grant C, Valentine A et al (1996). Brain glucose metabolism in chronic marijuana users at baseline and during marijuana intoxication. Psychiatry Res 67: 29-38.

Wechsler D (1999). Manual for the Wechsler Abbreviated Scale of Intelligence. The Psychological Corporation: San Antonio, TX.

Wetherill RR, Jagannathan K, Hager N, Childress AR, Rao H, Franklin TR (2015). Cannabis, cigarettes, and their co-occurring use: disentangling differences in gray matter volume. Int $J$ Neuropsychopharmacol 18: pyv061.

Xu F, Ge Y, Lu H (2009). Noninvasive quantification of whole-brain cerebral metabolic rate of oxygen (CMRO2) by MRI. Magn Reson Med 62: 141-148. 
$\mathrm{Xu} \mathrm{F}$, Uh J, Liu P, Lu H (2012). On improving the speed and reliability of T2-relaxation-under-spin-tagging (TRUST) MRI. Magn Reson Med 68: 198-204.

Yip SW, DeVito EE, Kober H, Worhunsky PD, Carroll KM, Potenza MN (2014). Pretreatment measures of brain structure and reward-processing brain function in cannabis dependence: an exploratory study of relationships with abstinence during behavioral treatment. Drug Alcohol Depend 140: $33-41$.

Zwillich CW, Doekel R, Hammill S, Weil JV (1978). The effects of smoked marijuana on metabolism and respiratory control. Am Rev Respir Dis 118: 885-891. (c) (1) (2) (2) This work is licensed under a Creative Commons Attribution-NonCommercial-ShareAlike

International License. The images or other third party material in this article are included in the article's Creative Commons license, unless indicated otherwise in the credit line; if the material is not included under the Creative Commons license, users will need to obtain permission from the license holder to reproduce the material. To view a copy of this license, visit http://creativecommons.org/licenses/by-nc-sa/4.0/

(C) The Author(s) 2018 